\title{
THE INTER-RELATIONSHIP BETWEEN INDOOR AND OUTDOOR POLLUTION IN SOUTH AFRICA IS IT A DILEMMA?
}

\author{
PRESENTED AT THE 72ND MEETING OF THE NATAL BRANCH OF NACA \\ ON WEDNESDAY APRIL 1, 1987 IN DURBAN
}

WILLEM BARWISE

The theme of this presentation is the inter-relationship between indoor and outdoor pollution in South Africa with special emphasis on the regulatory control of the different types of pollutants in this context by the authorities as well as the possible influence there of on industrialists.

To start off, let's attempt to define the "indoor" and "outdoor" pollution and to clarify the differences.

\section{(i) Pollution:}

The term pollution which is common to both concepts could be defined as follows:

The presence of any material, substance or energy not normally found in a medium whether this medium be solid, liquid or gaseous.

(ii) "Indoor" pollution refers to the pollution of the working environment and is encapsulated by the discipline of Occupational or Industrial Hygiene which defines it as the science (and art) devoted to the anticipation, recognition, evaluation and control of the environmental factors and stresses arising in and from the workplace which may cause illness, impaired health and well-being or significant discomfort and inefficiency among workers or among citizens of the community. In summary:

"To protect man from the environment and the environment from man."

From this definition it is obvious that not only the environment between four walls is examined, but also that which is emitted from a particular source that might have an influence on the surrounding areas.

(iii) "Outdoor" pollution in the context, refers to pollution of the non-working environment, or environmental pollution. In other words, residential and agricultural and other areas surrounding a plant, factory or source and includes not only air pollution but also water and surface pollution.

In both cases the prime factor to be considered is the influence of pollution on the human element and the effects thereof not only on mans' physiological, but also his psychological well-being and health.
Now lets digress a little and look at where it all started. In the Beginning man was created to breathe clean air and to live in total harmony with nature, free from contamination and pollution. We all know, however, the history of The Beginning; how man and his wife were thrown out of paradise to earn a living through hard labour and in the sweat of their brows. We in fact tonight are discussing one of the results of the sins of our fathers.

Although in those early days there was very little if any pollution due to the nomadic existence of the people, life was not entirely hazard free.

Things as far as pollution goes, however, changed drastically with the advent of the industrial revolution between 1760 1830. Suddenly man was taken from his nomadic existence in life and nature and put into an artificially created microenvironment contaminated by pollutants of all types resulting in, until then, unknown stresses, discomfort, ill health and even death. In many instances exposure to pollutants didn't stop for those men, women and children who slaved in the factories and mines of those days, but continued after-hours at home.

Home in many instances was the factory in which they worked during the day or was never too far from it as they had but a few hours to rest. Air pollution control in factories was non-existent and we all know what the results were, not even so very long ago.

Obviously, it didn't take too long for these working and living conditions to start taking their toll in the form of ill health and premature death.

As with all such occurrences, no or very little action was taken to prevent the exposure of people and the contamination of the environment, with the resultant adverse effects, until it was almost too late. Luckily there were individuals who started to fight for the workers to protect them from unsafe conditions in the workplace and who cared about the environment.

In 1835 the first Child Labour Law was passed in America prohibiting the employment of children under the age of 10 years in mines and factories. A special police officer to enforce this law was however only appointed 32 years later, in 1867. 
In Britain and elsewhere in the world the first observations, as early as 1493, by Paracelsus and Agricola were made on the diseases in mines. The first study on the diseases related to occupations, was made by Ramazzini between 1633 and 1714 .

Slavery was abolished in 1833 and in the same year the Factory Bill saw the light in Britain, bringing about the appointment of an inspectorate and a stipulation that a child must be certified by a doctor to be over the age of 9 years to be allowed to work in a textile mill.

Closer to home the first legislation promulgated by parliament to protect life and limb of mine workers, appeared in 1883 followed much later by the Mines and Works Act (Act 13 of 1911), the Factories Act (Act 28 of 1918), the Atmospheric Pollution Act (Act 45 of 1965) and the Machinery and Occupational Safety Act, (Act 6 of 1983) and many others.

This struggle to provide a clean and healthy working environment and to conserve and protect nature from the destructive creations of man, still continues today. There is no single answer available to solve all problems and the control of pollution, like many other things in nature, has to follow an evolutionary path. Legislation with teeth has long been recognised as the only tool to control pollution, as it is impossible to rely on the moral obligations of industry in an economic field.

The question that now arises is how to implement legislation so as to not only satisfy the worker in a factory or the citizens of the community, but also the industrialist and employer.

When we consider the present situation in South Africa I think you will agree that it could be somewhat confusing, for instance:

1. The internal working environment, or occupational hygiene field, falls under the jurisdiction of the Department of Manpower and their lever to control occupational hazards is the MOS Act. This act requires inter-alia that the employer must provide a safe and healthy working environment and be able to prove to an inspector that such an area is in fact safe.

The occupational hygiene hazards and stresses they consider include chemical, physical, ergonomical and biological and should also include physiological factors. Machine safety and accident prevention also resort under this department.

In order to enforce the regulations laid down, three different inspectorates exist namely; Machine Safety inspectors, Occupational Hygiene (Safety) Inspectors and also, in certain areas local authority health inspectors indirectly controlled by the Department of National Health, but appointed by the Department of Manpower to carry out certain inspections.

A draft Occupational Medicine Bill, also concerned with the occupational environment recently saw the light and would have been administered by the Department of National Health with probably their own inspectorate, but was subsequently shelved.

2. Whatever pollution leaves a factory through stacks and chimneys falls under the jurisdiction of the Department of National Health, with their tool of enforcement, the Atmospheric Pollution Prevention Act, applying the Best Practicable Means principle of control.

In the enforcement of the Act, local authority inspectors assist as far as non scheduled processes are concerned and together they look at aspects including dust, gas and smoke emissions from sources.

3. Local authority inspectors as mentioned earlier, are further involved in the control of community pollution eg. odours from a factory, excessive or disturbing noise, whether by industry, or a barking dog. They are also responsible for the control of motor vehicle exhaust gas emissions.

4. Then there is a grey area namely, the control of fugitive emissions from industrial buildings. I'm not sure whose responsibility this is as Department of Manpower feels that it is not their concern once a pollutant leaves a building and Department of Health could argue that it is not emitted from a stack or chimney.

Admittedly, what goes up must come down and Environmental Air Pollution Control, which also resorts under the jurisdiction of the Department of National Health, will eventually take action.

It is my belief that fugitive emissions today in our densely industrialised areas, are the biggest single source of poor air quality and that very special and urgent attention should be paid to the control thereof.

We have only concentrated on occupational hygiene and air pollution, but there are also other types of pollution eg. water pollution, with their own Acts, own Government departments and inspectorates to be considered.

The question that now arises is, why are all these different departments and inspectorates necessary for 
the control of pollution, both occupationally and environmentally? The results or effects, in a greater or lesser degree, are the same for all types of pollution whether experienced occupationally or not. It is thus normally only the degree of exposure that differs eg. higher concentrations of gases and dusts, prevail indoors than in the environment. As far as human exposure to many air pollutants is concerned, the end results, however, are normally the same eg. Asbestosis, mesothelioma, silicoses, etc., to name but a few conditions, whether these be contracted occupationally or environmentally, should the normal homeostatic capacity of the body be exceeded.

Monitoring techniques and principles applied for the quantification of these hazards are principally the same. For instance:

Evaluation of dusts and gases:

(i) In the Occupational Environment:

The methods normally applied are:

(a) Personal exposure monitoring for either total or respirable dust fractions or for gases, mists and vapours by cumulative or integrated sampling followed by chemical or other analysis.

(b) Strategic sampling by means of high volume sampling or direct reading or sensing instrumentation with, where necessary, relevant analysis.

\section{(ii) Environmental Monitoring:}

Personal exposure monitoring is not normally done, but the same equipment in certain instances could be used for both internal and external monitoring. Due to the dilution factors involved, direct sensing techniques for gases in the ppb range and integrated samples for dusts eg. by high volume and fallout sampling is done. As for occupational monitoring certain analyses of the samples will be required.

In both the above instances it is of the utmost importance to link the monitoring and results obtained with climatic or meteorological data eg. wind speed and direction, precipitation, temperature etc. as these parameters can have a marked and major influence on results and the interpretation thereof.

\section{(iii) Source monitoring:}

If the source is well defined eg. a stack or a fan outlet, the emission rates or concentrations of pollutants, based on direct sensing techniques, cumulative isokinetic sampling and subsequent analysis could be applied. Admittedly, source emission monitoring is not as simple as it sounds and instead of meteorological parameters flue gas data needs to be considered eg. gas temperatures, pressures, velocity etc. When emission data is integrated with meteorological parameters and topography, computer models could be used to predict contaminant concentrations for various climatic conditions at different distances from the source.

The monitoring or quantification of fugitives emitted from a building is very difficult if not virtually impossible and will, as previously mentioned, only be assessed once it reaches ground level.

Regarding the physical control of pollutants, the same basic principles again apply whether for occupational or environmental control purposes, and include the following: Dilution or General Ventilation, local exhaust systems with or without pollutant arrestors, isolation and/or substitution of harmful substances with less hazardous materials or combinations of the above.

\section{In summary then:}

Several different Government Departments and several inspection authorities presently control the same thing, namely pollution. An industrialist can face the prospect of receiving a visit from three or four different inspectors all concerned with the pollution problem - either Machine Safety, Occupational Safety, AirPollution control, Water pollution, Solid waste management, Odours, etc.

I am not suggesting that the individual Departments and inspectorates are not doing their jobs as best they can with the limited resources of manpower, funds and equipment available to them. The question I'd like to pose is, do we really need an array of Authorities such as now exists to control basically the same thing?

Will it not be far more economical and practical to have a single body, whether private or governmental, to control pollution in its' totality in South Africa?

I'm convinced that by establishing such a body or department without the in-fighting and interdepartmental jealousy that from time to time arises, the control of pollution in S.A. could be done on a far better, co-ordinated basis. Immediate implications would be that the country at large could be saved millions of Rands annually. 